\section{Cavity solitons in a}

\section{VCSEL: reconfigurable}

\section{micropixel arrays}

S. Barland ${ }^{1,2}$, M. Brambilla ${ }^{3}$, L. Columbo ${ }^{3}$, L. Furfaro ${ }^{\prime}$,

M. Giudici $i^{1}$, X. Hachair ${ }^{1}$, R. Kheradmand ${ }^{4,5}$, L.A. Lugiato ${ }^{4}$,

T. Maggipinto ${ }^{3}$, G. Tissoni ${ }^{4}$ and J. Tredicce ${ }^{1}$

${ }^{1}$ Institut Non Lineaire de Nice, France

${ }^{2}$ Istituto Nazionale di Ottica Applicata, Florence, Italy

${ }^{3}$ INFM and Dipartimento di Fisica Interateneo, Politecnico di

Bari, Italy

${ }^{4}$ INFM and Dipartimento di Scienze, Università dell'Insubria, Como, Italy

${ }^{5}$ Center for Applied Physics and Astronomical Research,

University of Tabriz, Iran

\section{Encoding optical information in space}

$F^{\text {or quite a few years, scientific and technological advances have }}$ $F$ made commonplace the possibility of encoding information in the modulation of light beams. Several techniques have been investigated to optimise such methods in the temporal domain or in its dual counterpart, i.e. in the radiation spectrum, with a procedure similar to encoding information in our voice. On the other side, encoding information in space is an everyday task we accomplish, with a wealth of technical means, when we "write". It all amounts to getting control of a homogeneous support, and breaking its translational symmetry with suitable procedures and tools (e.g. a pen on a sheet of paper, a photographic impression on a photosensitive surface).

Light, and particularly coherent, highly directional and controllable beams are suitable to encode information in space, especially due to the intrinsic parallelism of linear wave-like propagation. In optics, a widely exploited way to encode information in space makes use of arrays of micropixels, each emitting or transmitting a bright spot (see Box 1a). The price to pay of course is the rigidity of the array wherein the "units" are arranged, similar to what we have when we use a typewriter. Rigidity is a limitation indeed: beyond handwriting, educators make use of an even more flexible support, the blackboard, where writing/erasing, changing the layout of the encoded information etc. is even easier than with ink and paper. One might even dream of a blackboard where you can grasp an already jotted formula and "carry" it across a couple of panels without destroying it or what else you already wrote. To overcome the rigidity constraint, one may think of using as homogeneous support (blackboard) the transverse cross-section of a single broad area laser beam. However, in most laser beams the transverse configuration corresponds to a spatial mode of the laser cavity, whose parts are all correlated in space, and you cannot "act" to break its symmetry in one side, without having some other place in the beam changing its intensity profile in an unwanted manner. What we need is to find a "sign", an elementary structure of light that can be used as a minimal alphabet, can be "written" and "erased" and remains independent of what happens to the rest of the radiation profile, no matter what we do somewhere else in the homogeneous support.
A concept that comes to mind in order to realise this situation is that of spatial solitons (see Box 2), i.e. light beams which propagate without changing their transverse profile [1]. The element we miss in this case is, however, the persistence of the written "symbol". It can be transmitted to a receiver, or even forwarded to a downhill processing stage, but it does not remain where it has been "written".

In this paper we focus on a special class of soliton-like structures which occur in optical systems with cavities, where the feedback action of the mirrors make the elementary structure for encoding information (a bright light spot) a stationary, persistent solution of the device emission profile.

\section{Cavity Solitons as self-organised pixels}

It is well known since the late 80 s [2] that an optical system can spontaneously quit emitting a homogeneous field profile and form spatial patterns in the intensity profile, as it happens in hydrodynamics and other fields of science where spatially extended systems come into play. Optical pattern formation is similar to the formation of convective rolls in fluids or spiral waves in nonlinear chemical reactions. While there the spatial coupling occurs via convection and diffusion, in optics the basic mechanism is diffraction. Such phenomena were predicted and experimentally observed in several classes of optical systems [3].

When this occurs in a nonlinear resonator, the pattern forming instability emerges from the coupling of the nonlinear medium response, diffraction, and the dissipative/feedback action of the mirrors. The system loses its translational symmetry, and as long as its new spatially modulated phase is sustained by some form of energy intake, the optical system will keep on emitting a pattern which may be a regular lattice such as, for example, a hexagonal aggregate of bright light peaks or a honeycomb lattice (see Fig. 1). As such, this "global" structure is not a proper means to encode information: the pattern is a highly correlated structure, similar to a transverse mode, even if it is boundary independent. The light spots are there, but they are not individually addressable.

Nevertheless, this problem can be circumvented by realising a situation that, in the general field of pattern formation, is called "localised structure" [4]. This phenomenon generally arises under conditions of coexistence between two stationary states, one of which is homogeneous in space, the other patterned. As it happens, under appropriate conditions it is possible that a small portion of the pattern is embedded in a homogeneous background corresponding to the homogeneous profile. If the pattern is a hexagonal lattice one can try to reduce the modulated "island" coexisting with the homogeneous emission to its minimal ele-

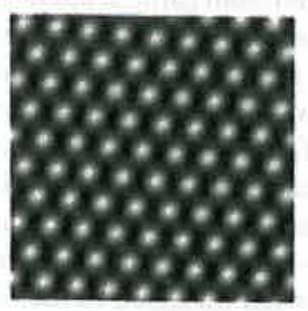

(a)

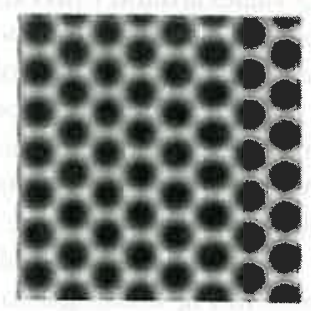

(b)
A Fig. 1: Numerical simulation for an optical nonlinear resonator showing (a) a hexagonal pattern, (b) a honeycomb pattern in the field intensity profile. 


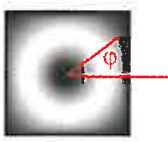

(a)

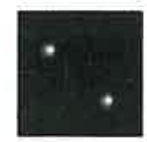

(b)

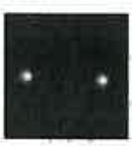

(c)

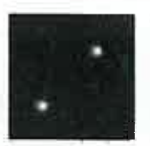

(d)

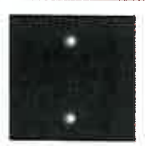

(e)
4 Fig. 2: Two cavity solitons perform a uniform rotary motion (b)-(e), along the crater of a doughnut-shaped holding beam (a), under the action of the angular gradient (numerical simulation).

ment, namely the single dots of the lattice. In the optical case, one will thus have a bright intensity maximum isolated in the background, and it will show no correlation whatsoever with its surroundings up to a minimal distance which is generally comparable to its own diameter [5]. This is what we call a Cavity Soliton (CS) and it can be realised immediately that it bears several substantial differences from the usual concept of a spatial soliton: for example, CSs may arise and be stable even in the presence of a self-defocusing nonlinearity. CSs can be individually written and erased by shining suitable laser pulses into the optical cavity containing the nonlinear medium (see Box lb).

An additional bonus of CSs is their capability of drifting across the transverse section of the optical system under the action of phase or amplitude gradients in the holding beam. CSs experience an Aristotelian force: their speed is proportional to the force, which in this case is created by the gradient [6]. In particular, the maxima of the phase/amplitude profile represent equilibrium positions for the CS.

In this way, CSs can be set in motion in a controlled manner. Figure 2 shows the case of a holding beam that has the shape of a doughnut mode (Fig. 2a). Two CSs exhibit a circular motion along the ring-shaped region where the intensity of the doughnut mode (which is shaped like the crater of a volcano) is maximal. The motion is induced by the fact that the phase of the doughnut mode varies as $\exp ( \pm i \varphi$ ), where (is the angle (see Fig. 2a), which creates a constant phase gradient along the crater, and the direction of motion is determined by the \pm sign.

If, instead, one tailors the holding beam in such a way that it displays a periodic phase modulation (Fig. 3a), this constitutes the immaterial support for an array of CS pixels (Fig. 3b,c and d), which can be individually set on and off by shining laser pulses. By varying the phase landscape, one can reconfigure the CS array, and by suitably introducing further gradients CSs can be brought to controlled interactions. It is certainly an attractive feature to make more and more flexible the all-optical stage of some optoelectronic devices which are presently employed such as smart pixel arrays and serial/parallel converters.

The possibility of controlling CS motion can be exploited for practical applications. In addition to reconfigurable optical memories and serial-to-parallel converters, examples of possible future applications are signal amplification, realisation of cellular automata, pattern recognition and optical tweezers.

A review of the fundamental and applicative features of CSs is though outside the scope of this contribution and the reader is referred to the bibliography (see [7] and references quoted therein).

\section{Cavity solitons in semiconductor microresonators: experimental demonstration and theoretical interpretation}

Experimental observations of CSs have been achieved in the past in various nonlinear optical materials, e.g. in photorefractive resonators and lasers with saturable absorbers, as well as in other systems with feedback utilising liquid crystals or atomic vapours. In all these cases, however, the cavity was macroscopic and the media characterised by slow response times.

But unquestionably, credible applications in the fields of optoelectronics and photonics require the use of miniaturised devices and fast nonlinear materials, as can be obtained by using semiconductor microresonators. Theories for this configuration [8] had to consider fundamental physical mechanisms, peculiar to

\section{Box 1}

\section{Rigid vs plastic pixels:} etched microresonators and self-confined cavity solitons

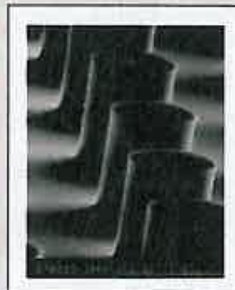

(a)

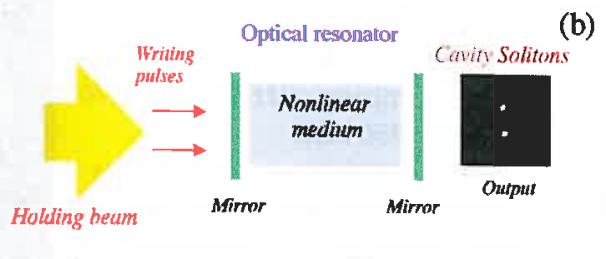

tence between the patterned and the homogeneous state. In order to create a CS, one adds a short and narrow "writing" pulse to the holding beam, aimed at a certain transverse point $(x, y)$. Provided that the pulse is in phase with the holding beam, its intensity locally increases the field and finally provides the system with the energy necessary to access the patterned branch. In the output intensity profile, one readily observes the formation of a bright peak. As the CS is a subset of a stable solution, when the pulse dies off, the peak indefinitely persists where it has been excited, as though the pulse drove a channel along the resonator axis, which becomes self-sustained by the mirrors' feedback action. It is of course straightforward to shoot more pulses at different locations of the transverse section of the system and turn on as many CS as the resulting distances between them will keep below the interaction range.

In order to switch a single CS off, with no consequences on the other CSs, it suffices to locally bring the system to a regime where the patterned state cannot persist any more. This is obtained by shooting at the location where a CS lies, an "erasing" pulse similar to the "writing" one but with opposite phase with respect to the holding beam. The local intensity thus decreases and the system precipitates to the homogeneous state, thus restoring homogeneity where the CS was. 


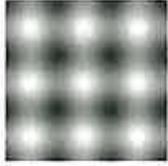

(a)

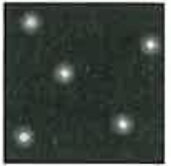

(b)

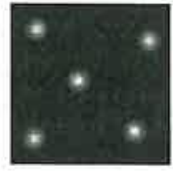

(c)

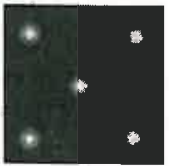

(d) $\triangle$ Fig. 3: In a periodic phase modulation (a), the maxima of the profile work as equilibrium sites for cavity solitons. (b), (c) and (d) illustrate the self-assembling behaviour of CSs; (b) Initial condition: five CSs are excited at some positions; (c) each CS is moving towards the nearest local phase maximum, (d) final condition: the CSs are placed at phase maxima of the array.

the semiconductor optical response and to the microresonator architecture.

From an experimental viewpoint, however, the realisation of CSs in semiconductor microcavities is a hard challenging task because of the small spatial and temporal scales, and the need for realising a broad area device with a reasonably uniform illumination and electrical pump current.

Although the phenomena of light localisation, i.e. candidates for CSs, had been reported [9], a clear-cut demonstration of objects that can be controlled independently of each other has been achieved only recently [10]. This goal was reached thanks to the close collaboration of four groups in the framework of the European ESPRIT Basic Research Project PIANOS (Processing of Information with Arrays of Nonlinear Optical Solitons). Experiments were carried out on a large number of samples, progressing in time towards optimisation of the device architecture, via a continuous interaction among the experimental (Nice), material (Ulm) and theoretical groups (Bari and Como). The device is a large area Vertical Cavity Surface Emitting Laser (bottom-emitter VCSEL, $150 \mu \mathrm{m}$ diameter) injected by a coherent field generated by a high power edge-emitter laser, whose wavelength is tunable in the range $960-980 \mathrm{~nm}$.

The VCSEL works as an amplifier and is kept slightly below the lasing threshold. A typical time-averaged transverse intensity profile of the VCSEL driven by the holding beam is shown in Fig. 4. One observes a homogeneous area on the right-hand side of the sample, and a patterned region on the left. As indicated by the numerical simulations, the most appropriate region to generate CSs lies immediately to the right of the line which separates the homogeneous and the patterned area.

\section{$30 \times 2$}

\section{Spatial Soliotions}

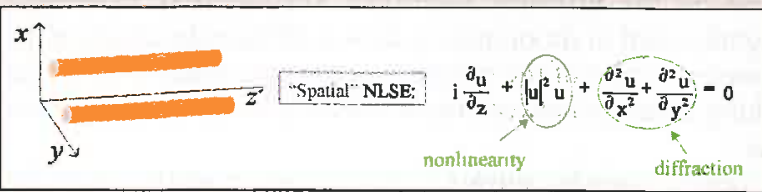

Spatial Solitons are light beams that propagate with undistorted shape, as a result of a perfect balance between diffraction, which tends to spread the beam, and the self-focusing action induced by the interaction with the nonlinear medium in which the beam propagates. In Kerr media they can be described by a Nonlinear Schroedinger Equation (NLSE).

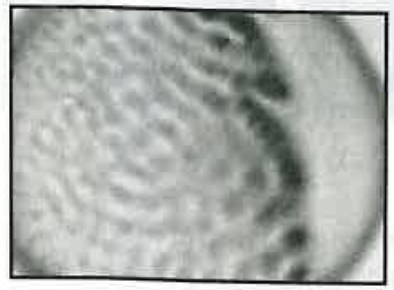

(a)

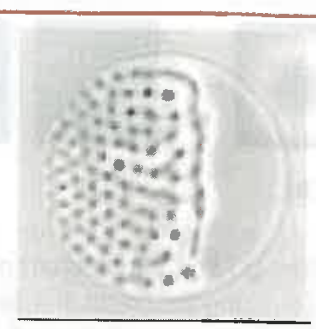

(b)
Aig. 4: Time averaged transverse intensity profile of a VCSEL driven by a coherent holding beam. (a) experiment, (b) numerical simulation. A patterned region appears on the left, a homogeneous domain on the right.

Starting with no spot, the writing beam (power about $50 \mu \mathrm{W}$, compared to $8 \mathrm{~mW}$ for the holding beam) is capable of generating a high intensity spot when it is in phase with the holding beam, as predicted by theory. When the writing beam is removed, the bright spot remains on indefinitely. After writing one spot, the writing beam is applied again in a different position, causing a second CS to turn on and persist after the writing beam is extinguished. This procedure has been improved with respect to the results reported in [10], where only two CSs were switched on, and presently up to 7 CSs can be independently excited in the sample (Fig. 5). This improvement is due to the introduction of a misalignment of the holding beam, so that the spatial region where the parameter values allow the existence of stable CSs is consequently enlarged.

As for turning the CS off, the writing beam must be aimed at an existing CS and its phase must be varied by $\pi$ so that when it is injected, it destructively interferes with the broad holding beam and locally depletes the sustaining field; this causes the CS to disappear, and when the writing beam is turned off, the spatial intensity distribution becomes exactly the same as it was at the beginning of the experiment.

The CSs in Fig. 5 can be controlled independently; however technical reasons due to the alignment precision of the writing beam do not allow all of them to be switched on and off: the erasing procedure works perfectly with 4 of the CS in Fig. 5, while three of them can only be switched on at present. This writing/erasing experiment was repeated at several values of the pumping current from 270 $\mathrm{mA}$ up to $350 \mathrm{~mA}$.

This unambiguous demonstration of CS stability and control in semiconductor microresonators opens up a broad applicative scenario for innovative optoelectronic devices based on CS properties. As an example, using the drifting properties of CS the fanning in and out of an optical signal could be much easier, as well as the

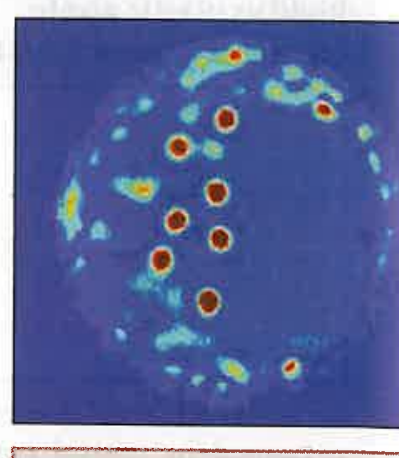

A Eio. S: Seven cavity solitons have been excited in the transverse plane by the injectio of a coherent writing beam. serial/parallel conversion. On the other hand, the problem of ensuring high levels of homogeneity and sample smoothness in the growing phase must be addressed in the future to achieve viable devices in photonics.

The most pressing short-term issue of this research involves a development of optical control techniques on the holding beam to ensure proper CS manipulation. 


\section{Acknowledgements}

This work was carried out in the framework of the ESPRIT Basic research Project PIANOS (Processing of Information by arrays of Nonlinear optical Solitons), PRIN project "Formazione e controllo di solitoni di cavità in microrisonatori a semiconduttore" of the Italian Ministry for University and Research, and the European Network VISTA (VCSELs for Information Society Technology Applications). R. Kheradmand undertook this work with the support of the ICTP Programme for Training and Research in Italian Laboratories, Trieste, Italy.

\section{References}

[1] R. Y. Chiao, in Springer Series in Optical Sciences Vol. 82, eds. S. Trillo and W. Torruellas, pp. 1-18 (Springer Verlag, 2001).

[2] L.A. Lugiato and R. Lefever, Phys. Rev. Lett. 58, 2209 (1987).

[3] F.T. Arecchi, S. Boccaletti and P. Ramazza, Phys. Rep. 318, 1 (1999); W.J. Firth in Self-Organization in Optical Systems and Applications in Information Technology, edited by M.A. Vorontsov and W. B. Miller, 69 (Springer Verlag, Berlin 1995); L.A. Lugiato, M. Brambilla and A. Gatti, in Advances in Atomic, Molecular and Optical Physics Vol. 40, eds. B. Bederson and H. Walther, pp. 229-306 (Academic Press, 1998).

[4] O. Thual and S. Fauve, J. Phys. 49, 1829 (1988).

[5] M. Brambilla, L.A. Lugiato and M. Stefani, Europhys. Lett. 34, 109 (1996).

[6] W.J. Firth and A.J. Scroggie, Phys. Rev. Lett. 76, 1623 (1996).

[7] W.J. Firth and G.K. Harkenss, in Springer Series in Optical Sciences Vol. 82, eds. S. Trillo and W. Torruellas, pp. 343-358 (Springer Velag, 2001).; L.A.Lugiato, Inroduction to the Special Issue on Cavity Solitons, IEEE J. Quant. Electron. 39, 193 (2003).
[8] M. Brambilla, L.A. Lugiato, F. Prati, L. Spinelli and W.J. Firth, Phys. Rev. Lett. 79, 2042 (1997); D. Michaelis, U. Peschel and F. Lederer, Phys. Rev. A 56, R3366 (1997).

[9] V.B. Taranenko, I. Ganne, R. Kuszelewicz and C. O. Weiss, Appl. Phys. B 72, 377 (2001).

[10] S. Barland, J.R. Tredicce, M. Brambilla, L.A. Lugiato, S. Balle, M. Giudici, T. Maggipinto, L. Spinelli, G. Tissoni, T. Knoedl, M. Miller and R. Jaeger, Nature 419, 699 (2002).

\section{About the authors}

Stephane Barland is presently a Post-doc with a contract of the Network VISTA of the European Community at the INOA in Florence. Massimo Brambilla is Associate Professor in Physics of Matter at the Polytechnic of Bari. Lorenzo Columbo is currently a Research Collaborator at the University of Bari. Xavier Hachair is a Ph.D. student at the INLN since 2001. Luca Furfaro is a Ph.D. student at the INLN since 2000. Massimo Giudici is Maitre de Conference at the Department of Physics, University of NiceSophia Antipolis. Reza Kheradmand is a Ph.D. student at the Tabriz University, Iran and an ICTP fellow at the University of Insubria in Como. Luigi A. Lugiato is full Professor of Quantum Electronics at the University of Insubria, and the Director of the INFM (Istituto Nazionale per la Fisica della Materia) Research Unit in Como. Tommaso Maggipinto is a research fellow at the University of Bari. Giovanna Tissoni is Researcher at the INFM Research Unit in Como. Jorge Tredicce is Professor of Physics at the University of Nice-Sophia Antipolis and Director of the INLN, CNRS. 